\title{
Spinal Cord Compression by Thoracic Vertebral Hemangioma-A Case Report
}

\author{
Peter Kalina \\ Department of Radiology, Mayo Clinic, Rochester, USA \\ Email: kalina.peter@mayo.edu
}

Received December 16, 2011; revised January 19, 2012; accepted January 30, 2012

\begin{abstract}
A 68 year old with lower extremity numbness, vibratory sensation loss, coldness and burning of his feet, unsteady gait, frequent falls and a sensory level had an MRI demonstrating a T7 and T8 vertebral body/posterior element lesion with epidural extension, cord compression and foraminal extension (Figures 1-5). Decompressive laminectomy/resection confirmed vertebral hemangioma, a common benign neoplasm that typically remains asymptomatic, found incidentally in $10 \%$ of the population. Progressive vertebral body hemangiomas may cause cord or nerve root compression due to epidural tumor extension, expanded bone, hematoma or fracture. Radiographs demonstrate course vertical striations caused by thick trabeculae. CT in indolent lesions demonstrates fat density while compressive lesions demonstrate soft tissue density. Indolent lesions follow fat signal on MRI; symptomatic lesions are T1 isointense/T2 hyperintense. Work-up for aggressive hemangiomas includes angiography to determine vascularity, identify feeding/draining vessels and identify blood supply to the cord. Biopsy helps differentiate hemangioma, lymphoma, myeloma or metastasis. Management of symptomatic hemangiomas includes vertebroplasty for pain, radiation for pain, compression or pre-op and decompressive laminectomy for epidural disease. Embolization of feeding vessels may be performed pre-op or may be curative. Hemangioma causing cord compression and neurologic symptoms by extraosseous extension is much less common than benign hemangioma. Imaging features may suggest potential for progression.
\end{abstract}

Keywords: Spinal Cord Compression; Vertebral Hemangioma

\section{Case Report}

A 68 year old male with a history of prostate cancer developed progressive lower extremity numbness and tingling, loss of lower extremity vibratory sensation, coldness and burning of his feet, unsteady gait and frequent falls. He had a sensory level just above the umbilicus. MRI was obtained (Figures 1-5). Sagittal T1 weighted images (Figure 1) demonstrated a heterogeneous signal lesion with hyperintensity of the osseous component and isointensity of the soft tissue component involving the T7 and $\mathrm{T} 8$ vertebral bodies and posterior elements with T6-9 epidural extension, significant T7 and T8 spinal cord displacement and compression as well as right T6-7, T7-8 and T8-9 neural foraminal extension. T2 weighted images demonstrate both the osseous and soft tissue components to be hyperintense (Figure 2). Axial T2 weighted images demonstrated significant spinal cord displacement and compression (Figure 3). Post contrast sagittal T1 demonstrated intense homogeneous enhancement (Figure 4). Post contrast axial T1 demonstrated intense Homogeneous enhancement (Figure 5). As a result of these MRI findings, considered together with his rapidly progressive neurologic deterioration and question of metas
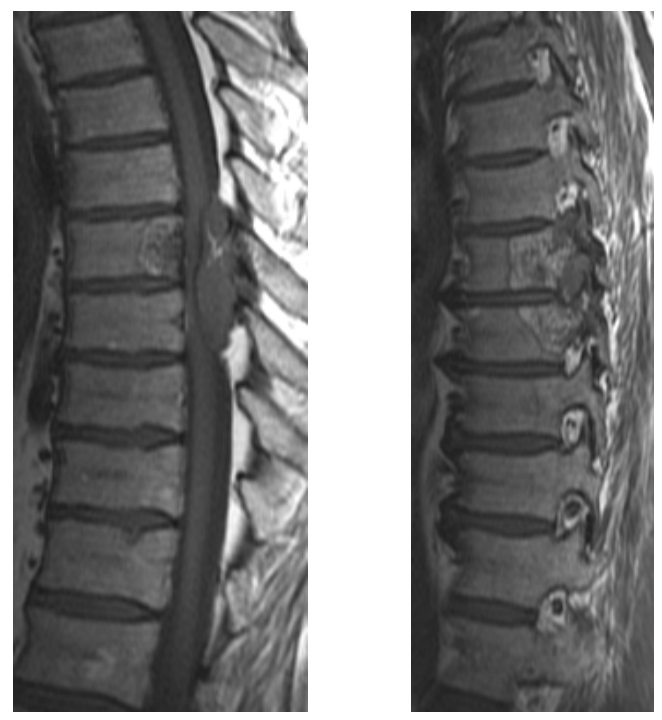

Figure 1. Sagittal T1 weighted MRI demonstrates a lesion of heterogeneous signal with hyperintensity of the osseous component and isointensity of the soft tissue component involving the $T 7$ and $T 8$ vertebral bodies and posterior elements with T6-9 epidural extension, significant T7 and T8 spinal cord displacement and compression as well as right T6-7, T7-8 and T8-9 neural foraminal extension. 

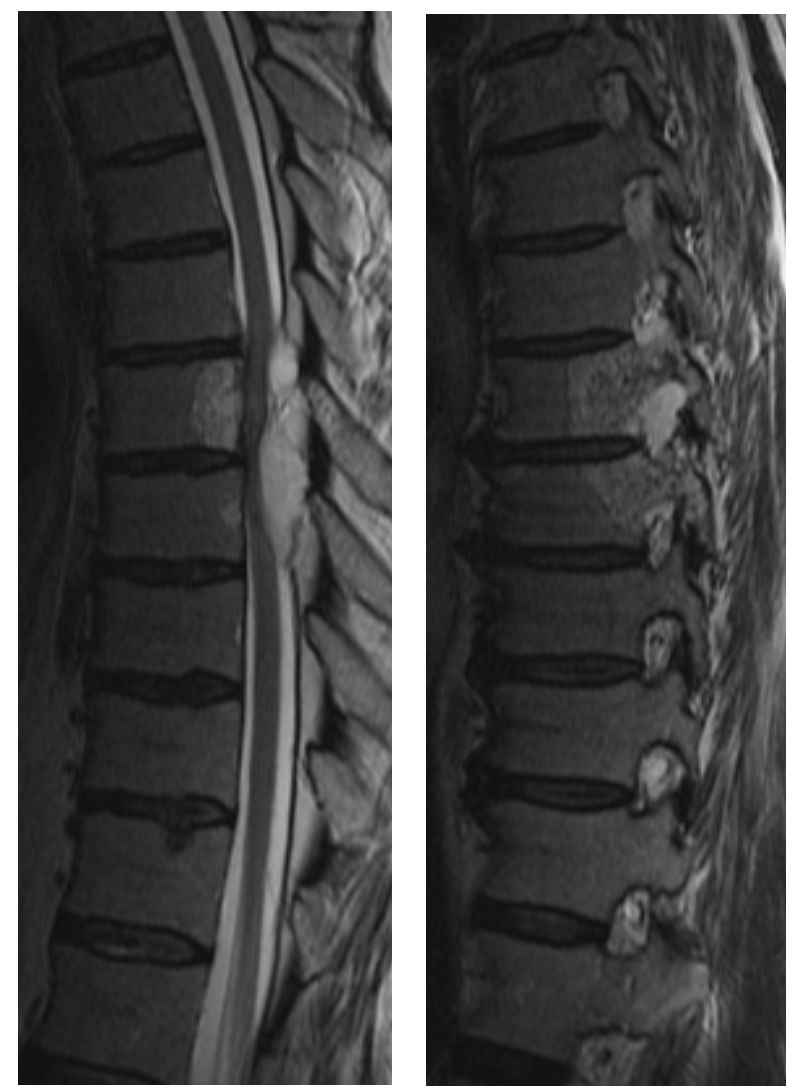

Figure 2. T2 weighted images demonstrate both the osseous and soft tissue components to be hyperintense.

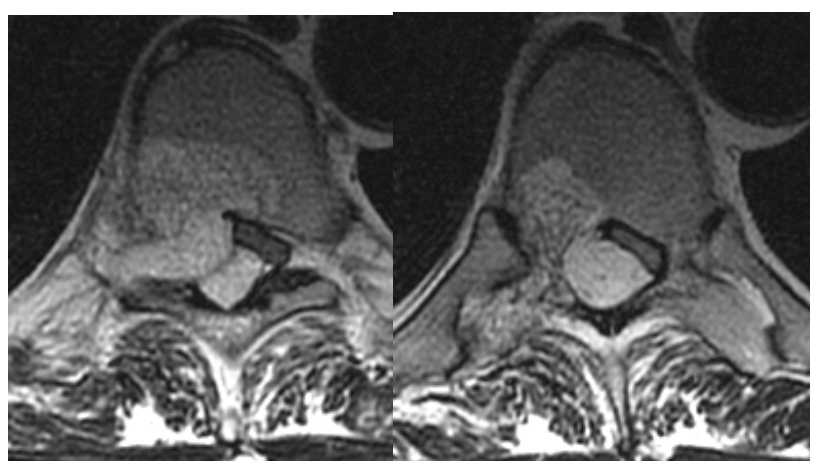

Figure 3. Axial T2 weighted images demonstrate the significant spinal cord displacement and compression.

tasis, decompressive laminectomy with partial resection was performed. Capillary hemangioma was confirmed at pathology. With the benefit of postoperative radiation, a significant recovery was possible.

\section{Discussion}

Vertebral hemangioma is a common benign neoplasm that typically remains asymptomatic throughout life, infrequently causing local or radicular pain or neurologic deficits. It is a common incidental finding seen in ap-
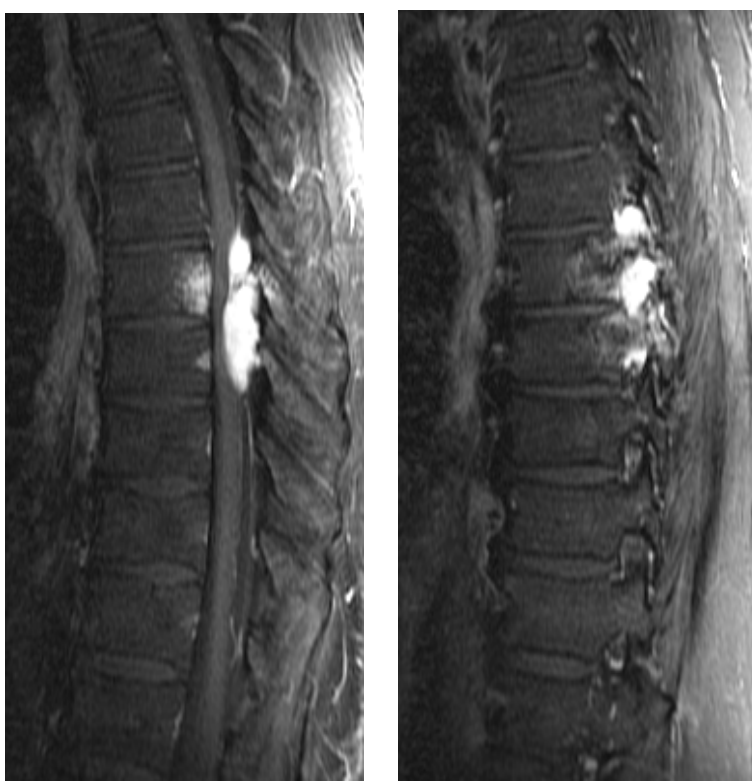

Figure 4. Post contrast sagittal T1 with fat saturation demonstrates intense homogeneous enhancement.

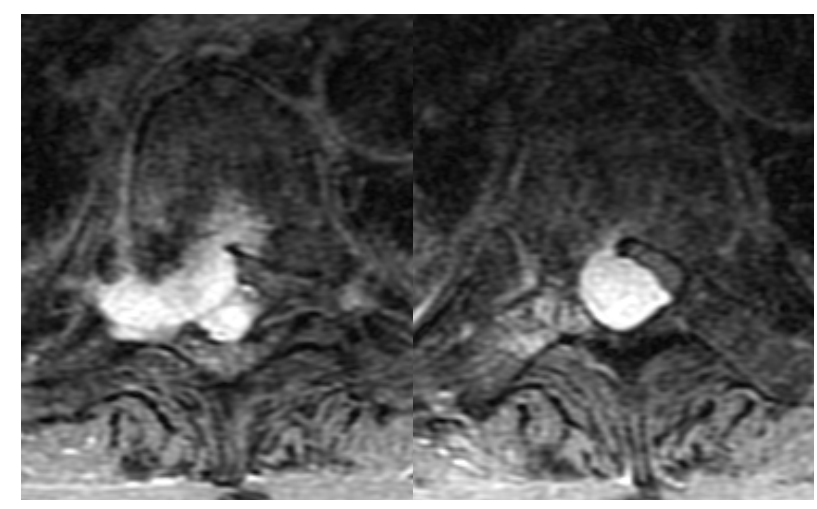

Figure 5. Post contrast axial T1 with fat saturation demonstrates intense homogeneous enhancement.

proximately $10 \%$ of the population [1]. It is more common in the thoracic region than in the lumbar or cervical regions. Multi-level involvement may occur. It affects the vertebral body more often than the posterior elements. Progressive vertebral body hemangiomas are those that are initially confined to the vertebral body, progress to involve the posterior arch and then expand further. Entire vertebra are involved by these progressive lesions more commonly than the vertebral body or posterior arch alone. Single level involvement is much more common than involvement of two or more contiguous levels. Pregnancy is a risk factor for the development of neurological symptoms in quiescent hemangiomas, possibly due to increased estrogen or increased flow in the vertebral venous system [1]. Cord or nerve root compression may be due to epidural tumor extension, expanded bone, hematoma, compression fracture or anomalous vessels. Cord compression is often progressive but may be sudden. 
Pain often precedes neurological symptoms, most commonly thoracic myelopathy. Imaging findings have been well characterized [2,3] Radiographs demonstrate course vertical striations caused by thick trabeculae. CT in indolent or inactive lesions demonstrates fat density while in symptomatic compressive lesions CT will demonstrate soft tissue density. Indolent lesions tend to follow fat signal on MRI while symptomatic lesions are $\mathrm{T} 1$ isointense and T2 hyperintense.

Extraosseous tumor contains very little fat although may demonstrate flow voids. Both types typically enhance. Pathology reveals hamartomatous proliferation of endothelial vascular tissue with secondary resorption of underlying bone. Most trabeculae are atrophic due to the abnormal blood vessels although some become thickened and sclerotic. Cavernous hemangiomas have multiple large thin walled vascular spaces while capillary hemangiomas have multiple capillary channels separated by reactive fibrous tissue [4]. Work-up for aggressive hemangiomas may include angiography to determine vascularity, identify feeding and draining vessels and identify the blood supply to the cord. CT guided biopsy may be warranted to differentiate hemangioma, lymphoma, myeloma or metastasis. Management of symptomatic vertebral hemangiomas can be very variable [5]. Vertebroplasty is contemplated for patients with localized pain. Radiation has been utilized for those with pain, pain and compression or pre-operatively. Post-op radiation may reduce the recurrence risk in subtotal tumor removal. Decompressive laminectomy is considered for resection of epidural disease. Embolization of feeding vessels may be a preoperative measure or may be curative. However it is not always necessary or possible if the feeding vessel also supplies the anterior spinal artery. Ethanol embolization has also been utilized. Compressive vertebral hemangioma causing cord compression and neurologic symptoms by extraosseous extension is much less common than benign hemangioma. The CT and MR features may suggest the potential for progresssion. An asymptomatic incidental hemangioma does not need further evaluation unless pain or neurological deficit develops at the appropriate level.

\section{REFERENCES}

[1] Y. Kiroglu, B. Benek, B. Yagci, et al., "Spinal Cord Compression Caused by Vertebral Hemangioma Being Symptomatic during Pregnancy," Surgical Neurology, Vol. 71, No. 4, 2009, pp. 487-492. doi:10.1016/j.surneu.2007.09.025

[2] S. Nassar, F. Hanbali, M. Haddad and M. Fahl, “Thoracic Vertebral Hemangioma with Extradural Extension and Spinal Cord Compression," Clinical Imaging, Vol. 22, No. 1, 1996, pp. 65-68. doi:10.1016/S0899-7071(97)00068-5

[3] C. R. Templin, J. B. Stambough and J. L. Stambough, "Acute Spinal Cord Compression Caused by Vertebral Hemangioma,” The Spine Journal, Vol. 4, No. 5, 2004, pp. 595-600. doi:10.1016/j.spinee.2003.08.034

[4] J. Rich, T. Donahue and T. Mick, "Symptomatic Expansile Vertebral Hemangioma Causing Conus Medullaris Compression,” Journal of Manipulative and Physiological Therapeutics, Vol. 28, No. 3, 2005, pp. 194-198. doi:10.1016/j.jmpt.2005.02.017

[5] P. Singh, N. Mishra, H. Dash, et al., "Treatment of Vertebral Hemangioma with Absolute Alcohol Embolization, Cord Decompression, and Single Level Intstrumentation,” Neurosurgery, Vol. 68, No. 1, 2011, pp. 78-84. doi:10.1227/NEU.0b013e3181fc60e9 